\title{
Pérou. Le Projet éducatif national Amérique latine
} 2021

\section{Adelaïda Méndez Villegas}

Traducteur : Philippe Rabaté

\section{OpenEdition \\ Journals}

Édition électronique

URL : http://journals.openedition.org/ries/828

DOI : $10.4000 /$ ries.828

ISSN : 2261-4265

Éditeur

Centre international d'études pédagogiques

\section{Édition imprimée}

Date de publication : 1 septembre 2010

Pagination : 20-24

ISBN : 978-2-85420-582-4

ISSN : $1254-4590$

Référence électronique

Adelaïda Méndez Villegas, « Pérou. Le Projet éducatif national Amérique latine 2021 », Revue internationale d'éducation de Sèvres [En ligne], 54 I septembre 2010, mis en ligne le 01 septembre 2013, consulté le 14 novembre 2019. URL : http://journals.openedition.org/ries/828 ; DOI : 10.4000/ries.828

Ce document a été généré automatiquement le 14 novembre 2019.

(c) Tous droits réservés 


\title{
Pérou. Le Projet éducatif national Amérique latine 2021
}

\author{
Adelaïda Méndez Villegas \\ Traduction : Philippe Rabaté
}

L'auteur remercie Jean-Marie De Ketele, Pierre-Louis Gauthier et Philippe Rabaté.

1 De nombreux questionnements sur les politiques éducatives agitent aujourd'hui le Pérou. Des organisations d'éducateurs s'efforcent d'orienter l'éducation à partir des expériences personnelles et collectives que mènent les étudiants à l'école; la naissance d'une éducation de qualité fondée sur la formation de valeurs devient une nécessité impérieuse.

2 Après la réforme éducative qui débuta en 1970 sous l'impulsion du général Juan Velasco Alvarado et qui constituait, dans un contexte d'effervescence sociale, une occasion historique de changement stratégique, les gouvernements suivants optèrent pour une orientation radicalement différente. Ainsi, Alberto Fujimori instaura une réforme éducative inspirée par une vision néolibérale qui a dominé la politique péruvienne tout au long de ces vingt dernières années; elle fut marquée par un désintérêt pour la formation initiale et les conditions de travail des maîtres, souvent condamnés à percevoir deux salaires pour assurer leur subsistance. Malgré ce contexte défavorable, la société civile se manifesta dans les années quatre-vingts, notamment avec l'apparition des écoles productives du MANTHOC ${ }^{1}$.

\section{Le Projet éducatif national Amérique latine 2021 et ses premières réalisations*}

Durant le gouvernement provisoire de Paniagua, des composantes progressistes de la société péruvienne s'accordèrent sur la nécessité de pouvoir s'appuyer sur un Projet éducatif national (PEN). Afin de l'élaborer, on procéda à la nomination d'un Conseil national de l'éducation qui siégea entre 2002 et 2008. Le PEN fut officiellement proclamé politique d'État en 2007 durant le gouvernement d'Alan García. 
4 Le Projet éducatif national Amérique latine 2021, L'éducation que nous voulons pour le Pérou, est le fruit des nombreuses contributions reçues depuis la diffusion du document Vers un Projet éducatif national (2005). L'élaboration de ce projet a donné lieu à un débat et à des échanges nourris dans les vingt-six régions du pays qui se sont efforcées de l'articuler avec l'élaboration de leurs projets éducatifs régionaux respectifs. Des professeurs, des représentants régionaux de l'éducation et d'autres secteurs ainsi que des chefs d'entreprise, des professionnels de diverses spécialités, des leaders d'opinion, des jeunes, des dirigeants d'organisations syndicales et du monde de la culture ont participé à sa rédaction.

Le PEN offre une vision précise de l'éducation: "Tout être humain développe son potentiel depuis sa plus tendre enfance, accède ensuite au monde de la lecture et de l'écriture, résout des problèmes, pratique des valeurs, sait continuer à apprendre, s'assume comme citoyen, avec ses droits mais aussi ses responsabilités, et contribue au développement de sa communauté et de son pays en combinant son capital culturel et naturel avec les grandes avancées mondiales » (p. 11).

6 Cette vision repose concrètement sur six objectifs stratégiques : offrir des opportunités et des résultats éducatifs de qualité égale pour tous; permettre que les étudiants et les institutions disposent d'apprentissages pertinents et de qualité ; offrir aux enseignants une préparation de bon niveau pour qu'ils exercent de manière professionnelle leur métier ; promouvoir une gestion décentralisée, démocratique, financée avec équité et qui soit source de bons résultats; atteindre une éducation supérieure de qualité qui deviendra un atout majeur pour le développement et la compétitivité du pays; développer le modèle d'une société qui éduque ses citoyens et les pousse à s'engager dans la défense et le développement de leur propre communauté.

7 Il convient de souligner que ces objectifs s'intègrent dans le cadre des postulats d'organisations internationales comme le Projet régional d'éducation pour l'Amérique latine et les Caraïbes - PRELAC ${ }^{2}$ (La Havane, 2002) -, le Comité pour les droits de l'enfant des Nations-Unies ${ }^{3}$ et la XVIIIe Conférence ibéro-américaine d'éducation ${ }^{4}$ (Le Salvador, mai 2008). Lors de cette dernière rencontre, des objectifs en politique éducative pour 2021 avaient été soumis à l'ensemble des pays membres et avaient donné lieu à une position commune sur l'éducation souhaitée pour la génération du bicentenaire des indépendances ${ }^{5}$.

8 Suivant les grandes lignes du PEN, le ministère de l'éducation a développé depuis 2007 le Programme national de formation continue (PRONAFCAP ${ }^{6}$ ), qui tend à organiser et à développer les activités de formation et de spécialisation des professeurs en poste. Ces dernières doivent répondre aux exigences d'apprentissage formulées par les étudiants et la communauté à laquelle ils appartiennent, ainsi qu'aux besoins réels de formation des professeurs des institutions éducatives publiques de l'ensemble du pays. Les universités et instituts supérieurs pédagogiques disposant d'une expérience en formation initiale et continue assument la planification, l'organisation, l'exécution, l'évaluation et la divulgation de l'information pour le dispositif PRONAFCAP.

9 L'orientation méthodologique du programme s'enracine dans la réflexion de l'enseignant sur ses propres croyances et sa pratique éducative, et le pousse à les améliorer après avoir élargi et approfondi les connaissances et les stratégies d'enseignement de sa spécialité et 
sa maîtrise du programme scolaire. La formation se développe par le biais de cours théoriques et pratiques répartis en quatre composantes :

- communication : l'objectif principal est que l'enseignant comprenne des textes variés, en identifiant les idées principales et secondaires, en mettant en relation et en assimilant l'information, et en définissant le propos et le contenu du texte ;

- mathématiques : former les enseignants afin qu'ils résolvent des situations problématiques en appliquant des connaissances et des procédés mathématiques ;

- conception d'un programme national, aspects spécifiques par niveau et spécialité académique : avec des heures en présence et à distance ;

- conception d'un programme national, aspects généraux: pour offrir à l'enseignant la possibilité de planifier, d'exécuter et d'évaluer les processus pédagogiques en accord avec les caractéristiques, les besoins et le contexte des étudiants; parallèlement, des axes transversaux du processus de formation seront abordés : la compréhension de la lecture, la formation aux valeurs éthiques et l'éducation inclusive.

10 Pour 2009 et 2010, le PRONAFCAP conduit le Programme de spécialisation pour l'enseignement de communication et mathématique pour les professeurs des $2 \mathrm{e}$ et $3 \mathrm{e}$ cycles d'EBR ${ }^{7}$. Les enseignants de ces niveaux participent à des sessions de monitoring et de tutorat. Le monitoring consiste en un accompagnement périodique du participant par le formateur afin de recueillir toute l'information importante sur les forces et faiblesses de son action dans la classe. Le tutorat est un échange et un dialogue entre le participant et le formateur pour renforcer le développement des capacités personnelles, sociales et pédagogiques de l'enseignant.

\section{Tendances progressistes dans la pensée éducative péruvienne actuelle}

Cette réforme pourrait par conséquent constituer une véritable réponse à la situation de crise que connaît le système éducatif péruvien à travers la recherche de quatre résultats centraux : le développement de la quantité et de la couverture (les services éducatifs qui rendent concrets le droit à l'éducation doivent être accessibles à tous les Péruviens), la recherche de la qualité de l'éducation et de la pertinence des apprentissages (pertinence aussi bien sociale qu'identitaire, fondée sur l'environnement des étudiants) et, finalement, la promotion de l'équité avec une valorisation de tous les étudiants sans différence de genre ni de race.

En prenant en considération les politiques éducatives du moment et leur évolution possible pour les prochaines années sous l'influence des développements internationaux, de nombreux éducateurs progressistes proposent des actions concrètes qui constituent de véritables défis pour le système. Cusianovich (2007) s'intéresse à l'éducation des secteurs populaires, spécialement en ce qui concerne l'éducation des garçons et des filles et celle des adolescents qui travaillent. Chiroque (2009), pour sa part, insiste à la fois sur la dimension sociale de l'éducation dans la lutte contre la pauvreté - en développant le lien entre éducation et équité sociale -, sur le droit prioritaire des minorités à recevoir une éducation égalitaire et, enfin, sur la nécessité de privilégier une approche fondée sur la durabilité de nos ressources naturelles.

13 Ricardo Villanueva Valverde ${ }^{8}$ partage la plupart de ces objectifs et y ajoute celui du renforcement de la gouvernance, avec la participation des gouvernements régionaux et 
locaux dans la construction des infrastructures sociales ainsi que dans l'innovation et la technologie en fonction des demandes définies conjointement avec le secteur de la production. Pour Hugo Diaz ${ }^{9}$, il existe des compétences indispensables que l'éducation péruvienne devrait faire siennes: la communication, les mathématiques, le développement scientifique, la réponse aux besoins $\mathrm{du}$ monde $\mathrm{du}$ travail, l'épanouissement de la personne et du citoyen, dans un cadre qui favorise l'alliance entre le professeur et les familles et qui se fonde sur les nouvelles technologies. Enfin, Jaramillo (mars 2009) propose de « lancer une grande croisade et une mobilisation citoyenne dans les différentes couches de l'espace social [du] pays pour mener à bien les changements radicaux et révolutionnaires dont a besoin l'éducation péruvienne » (p. 30).

En somme, l'éducation péruvienne traverse une situation d'abandon, car elle n'a jamais été la préoccupation centrale du gouvernement. Si la conception du Projet éducatif national en 2007 a constitué un progrès, trois années se sont écoulées et on ne l'applique guère de manière systématique : les résultats sont donc peu visibles. Les pouvoirs locaux, régionaux et nationaux doivent s'engager non seulement afin d'administrer le système éducatif mais aussi, plus fondamentalement, afin d'œuvrer à une société éducatrice qui forme ses citoyens à l'exercice plein et entier de leurs droits, pour une coexistence sociale plus humaine, et afin d'assurer le développement plein et entier des zones rurales.

\section{BIBLIOGRAPHIE}

CHIROQUE Sigfredo (2009) : Perú 2010-2015. Escenarios posibles y educación, Inédito. Lima.

CUSIANOVICH Alejandro (2007) : Aprender la Condición Humana. Ensayo sobre pedagogía de la ternura. Ifejant, Lima.

Institución Educativa Monseñor Julio González Ruiz (2009) : Enfoque de Escuela Productiva de MANTHOC, documento de trabajo, Lima.

JARAMILLO Enrique (2008-2009) : Educación comparada. Trabajo de investigación: Plan Especial de Bachillerato (mayo 2008). P.U.C.P. Lima ; La educación peruana está en el sótano...! Y los ciudadanos, ¿qué estamos haciendo? Artículo inédito (marzo, 2009). Lima.

MENDEZ VILLEGAS Adelaida y ORIHUELA ORRILLO Ana María (2009) : Tendencias Educativas. En el marco de la Pro-puesta Pedagógica de MANTHOC. Inédito.

\section{NOTES}

1. Acronyme du Mouvement des Adolescents et Enfants Travailleurs, issus de familles ouvrières chrétiennes.

2. PRELAC : http://portal.unesco.org/geography/es/ev/ .

3. Convention sur les droits de l'enfant : http://www2.ohchr.org/ .

4. XVIII ${ }^{e}$ Conférence ibéro-américaine d'éducation : http://api.ning.com/files/. 
5. La célébration du bicentenaire de l'indépendance du Pérou (1821) viendra clore le cycle des manifestations en 2021.

6. PRONAFCAP 2009 : http://www.oei.es/pronafcap_2009/ .

7. Ce second cycle correspond à l'éducation des enfants de 5 ans tandis que le troisième cycle comprend les deux premières années de primaire.

8. Cité par Chiroque (2009) dans la rubrique « Défis actuels pour l'éducation péruvienne ».

9. Id.

INDEX

Mots-clés : réforme de l'enseignement, système scolaire

Index géographique : Pérou

\section{AUTEURS}

\section{ADELAÏDA MÉNDEZ VILLEGAS}

Adelaïda Méndez Villegas est accompagnatrice d'enseignants de la première et deuxième année primaire, dans le cadre du Programa Nacional de Capacitación y de Formación Docente du Pérou. Après des études de pédagogie, elle a été institutrice puis a émigré en Belgique, suite aux événements politiques dans son pays. Titulaire d'un DEA en sciences de l'éducation obtenu à l'Université catholique de Louvain et nationalisée belge, elle est rentrée au Pérou où elle suit de près l'évolution du système éducatif. Elle a publié des articles en Espagne, au Canada et en Suisse. 